\title{
Aorta-Derived Mesoangioblasts Can Be Differentiated into Functional Uterine Epithelium, but Not Prostatic Epithelium or Epidermis, by Instructive Mesenchymes
}

\author{
Liz Simon ${ }^{\mathrm{a}}$ Paul S. Cooke ${ }^{\mathrm{b}}$ Suzanne E. Berry ${ }^{\mathrm{c}-\mathrm{e}}$ \\ ${ }^{a}$ Department of Physiology, Louisiana State University Health Sciences Center, New Orleans, La., ${ }^{b}$ Department \\ of Physiological Sciences, University of Florida, Gainesville, Fla., and ' Department of Comparative Biosciences, \\ University of Illinois at Urbana-Champaign, and d Institute for Genomic Biology and eNeuroscience Program, \\ University of Illinois, Urbana, III., USA
}

\section{Key Words}

Ectoderm · Endoderm · Estrogen · Mesoderm · Tissue recombination · Uterus

\begin{abstract}
Mesoangiobasts are blood vessel-derived stem cells that differentiate into smooth, skeletal, and cardiac muscle cells. We have reported that postnatal aorta-derived mesoangioblasts (ADM) regenerate skeletal muscle and prevent onset of dilated cardiomyopathy in animal models of Duchenne muscular dystrophy. ADM also differentiate into myelinating glial cells, suggesting they are multipotent and capable of generating mesodermal or ectodermal derivatives. Mesenchyme of some fetal organs is a potent instructive inducer. Here we examined whether ADM can differentiate into prostatic, uterine, and skin epithelium by recombining ADM with fetal or neonatal mesenchyme from these organs and grafting them under the renal capsule of syngeneic hosts. In tissue recombinants of uterine mesenchyme (UtM) and ADM, ADM formed histologically normal simple columnar uterine epithelium that expressed estrogen receptor 1 and in response to estrogen showed increased mitogenesis and downregulation of progesterone receptor. In contrast, ADM did not differentiate into prostatic epithelium or epidermis when recombined with urogenital sinus mesen-
\end{abstract}

chyme or fetal dermis, respectively. These results indicate that ADM can respond to cues from neonatal UtM and differentiate into morphologically and functionally normal uterine epithelial cells, and support previous reports that ADM can differentiate into a variety of tissues of the mesodermal lineage. However, these data indicate that ADM are restricted in their capacity to differentiate into endodermal and ectodermal derivatives such as prostatic and skin epithelial cells, respectively.

(c) 2013 S. Karger AG, Basel

\begin{tabular}{ll}
\hline Abbreviations used in this paper \\
\hline ADM & $\begin{array}{l}\text { aorta-derived mesoangioblasts } \\
\text { cK-8 }\end{array}$ \\
cytokeratin 8 \\
$1,1^{\prime}$ dioctadecyl- $3,3,3^{\prime}, 3^{\prime}$ - \\
tetramethylindocarbocyanine perchlorate \\
E & $17 \beta$-estradiol \\
ESR1 & estrogen receptor 1 \\
GFP & green fluorescent protein \\
MKI67 & antigen identified by monoclonal antibody Ki67 \\
PBS & phosphate-buffered saline \\
PR & progesterone receptor \\
SSC & spermatogonial stem cells \\
UGE & urogenital sinus epithelium \\
UGM & urogenital sinus mesenchyme \\
UtM & uterine mesenchyme \\
UtE & uterine epithelium
\end{tabular}

\section{KARGER}

E-Mail karger@karger.com

www.karger.com/cto
(C) 2013 S. Karger AG, Basel

$1422-6405 / 13 / 1983-0169 \$ 38.00 / 0$
Prof. Suzanne E. Berry

Department of Comparative Biosciences, University of Illinois at Urbana-Champaign 2001 South Lincoln Avenue

Urbana, IL 61802 (USA)

E-Mail berryse@illinois.edu 


\section{Introduction}

We have derived mesoangioblast stem cells from postnatal aorta (aorta-derived mesoangioblasts, ADM) and demonstrated that ADM regenerate skeletal muscle [Berry et al., 2007] and stimulate endogenous cardiac stem cells to generate new cardiomyocytes in animal models of Duchenne muscular dystrophy [Chun et al., 2013]. Mesoangioblasts derived from dorsal aorta [Minasi et al., 2002], postnatal bone marrow [Palumbo et al., 2004], skeletal muscle [Morosetti et al., 2006; Sampaolesi et al., 2006], and cardiac muscle [Galvez et al., 2008], as well as ADM, have the potential to differentiate into mesodermal derivatives. The cell type most commonly derived from mesoangioblasts of different tissues is skeletal muscle. Mesoangioblasts were identified by De Angelis et al. [1999] during a search for embryonic tissue anlage that contained myogenic progenitors. In addition to differentiating into smooth, skeletal, and cardiac muscle, mesoangioblasts derived from embryonic dorsal aorta could also differentiate into other cell types of mesodermal origin, including osteoblasts, chondrocytes, adipocytes, and blood cells following transplantation into chick embryos [Minasi et al., 2002]. Using this approach, embryonic mesoangioblasts were not observed to differentiate into ectodermal or endodermal derivatives, and mesoangioblasts derived from most postnatal tissues have not been specifically tested for their ability to differentiate into cell types of endodermal or ectodermal origin in mammalian systems. However, we have reported in two separate studies that ADM expressed markers of myelinating glial precursors and can be induced to mature into Schwann cells or oligodendrocytes that myelinate axons in the peripheral and central nervous system, respectively [Berry et al., 2007; Wang et al., 2012]. This is consistent with a previous report that mesoangioblasts from dorsal aorta expressed markers of neural cells, although no method was identified for inducing embryonic mesoangioblasts to differentiate into the neural lineage [Tagliafico et al., 2004]. As a result of these studies, we hypothesized that aortic mesoangioblasts could give rise to ectodermal derivatives, in addition to mesodermal derivatives.

In this study, we used a well-characterized tissue recombination methodology to test the potential of ADM to differentiate into cell types derived from all three germ layers. This procedure has been used extensively to characterize stromal-epithelial interactions in male and female urogenital tract development [Cunha et al., 1987; Kurita et al., 2001]. Mesenchymal and epithelial cell layers from developing fetal or neonatal organs are enzy- matically isolated, recombined, and grafted beneath the renal capsule of syngeneic host mice. These tissue recombination assays have revealed the importance of inductive signaling between urogenital sinus (UGS) mesenchyme (UGM) and epithelium during prostatic bud formation [Cunha et al., 1987] and between uterine mesenchyme (UtM) and epithelium (UtE) during uterine development [Kurita et al., 2001]. In addition, we have previously used tissue recombination to examine the differentiation potential of spermatogonial stem cells (SSC) by recombining instructive mesenchyme from developing uterus, skin, or prostate with these stem cells and examining whether the mesenchyme induced SSC to differentiate into epithelium of endodermal, ectodermal, or mesodermal origin [Simon et al., 2009]. Here we have used this tissue recombination approach to test whether ADM can differentiate into tissue derived from all germ layers. Our results show that ADM can differentiate into UtE that expresses unique morphological and functional markers of this tissue, but do not appear to be capable of differentiating into epithelial cells of ectodermal or endodermal origin.

\section{Materials and Methods}

\section{Cell Culture}

ADM were isolated as previously described [Berry et al., 2007; Wang et al., 2012]. Briefly, aortic arches from 2-week-old C57Bl/6 pups were isolated from the point below the heart ventricles to the site of entry into the atrium. The innominate, left subclavian, and left common carotid arteries were excised from the arch. Explant cultures of the arch were grown on gelatin-coated dishes in Iscove's Dulbecco's modified Eagle's medium supplemented with $20 \%$ fetal bovine serum, $0.1 \mathrm{U} / \mathrm{ml}$ penicillin, $0.1 \mu \mathrm{g} / \mathrm{ml}$ streptomycin, $2.0 \mathrm{mM}$ L-glutamine, $0.1 \mathrm{~mm}$ nonessential amino acids, minimal essential medium vitamin solution (Gibco, Carlsbad, Calif., USA), and $20 \mathrm{ng} / \mathrm{ml}$ purified leukemia inhibitory factor (Chemicon International, Temecula, Calif., USA).

Two clonally derived populations of ADM, M25.2, and M2.1, that differentiate into smooth, skeletal, and cardiac muscle, preadipocytes, and oligodendrocytes in vitro and in vivo [Berry et al., 2007; Odintsov et al., 2011; Wang et al., 2012; Chun et al., 2013] were used in this study. These cell populations both divide indefinitely and maintain their stem cell marker profile for at least 40 population doublings in culture [Wang et al., 2012]. ADM at passage 20 were used for tissue recombinations in this study. The surface marker profile of M2.1 and M25.2 was determined using antibodies conjugated to fluorophores as previously described [Wang et al., 2012]. Briefly, live cells were pelleted, suspended in $100 \mu \mathrm{l}$ of phosphate-buffered saline (PBS), and labeled with primary antibodies conjugated with biotin for $30 \mathrm{~min}$ on ice. Primary antibodies used were rat MCA2183B monoclonal antibody to CD13 (AbD Serotec, Raleigh, N.C., USA), mouse monoclonal antibody to CD146 (Chemicon/Millipore, Billerica, Mass., USA), rat monoclo- 
nal antibody to CD34 (eBioscience, San Diego, Calif., USA), rat monoclonal antibody to Thy-1.2 (CD90.2; eBioscience), rat monoclonal antibody to CD45 (eBioscience), rat monoclonal antibody to Ly-6A/E (Sca-1; eBioscience), and rat monoclonal antibody to PE-CAM-1 (CD31; eBioscience). Cells were washed twice in PBS and subsequently incubated in $100 \mu \mathrm{l}$ of PBS containing secondary antibodies conjugated with streptavidin-ECD (a conjugate of PE and Texas red). Cells were incubated with secondary antibodies for 30 min on ice, then sorted with an iCyt Reflection cell sorter and percentages of cells expressing surface markers were determined using Reflection's WinList Acquisition and Analysis software (Verity Software House, Topsham, Me., USA.)

Stably transfected ADM [Chun et al., 2013] and ADM labeled with the lipophilic fluorescent dye DiI (1,1'-dioctadecyl-3,3,3', $3^{\prime}$ tetramethylindocarbocyanine perchlorate) or iron oxide nanoparticles were initially tested in the tissue recombination procedure as a means to distinguish ADM from cells derived from fetal mesenchyme. However, as a result of proliferation of DiI-labeled $\mathrm{ADM}$, the label was diluted to the point where ADM could not be detected. ADM derived from green fluorescent protein (GFP) mice silenced GFP over time in culture. Moreover, ADM stably transfected with GFP [Chun et al., 2013] did not have the same differentiation capacity in vitro and GFP- and iron-oxide nanoparticle-labeled ADM did not have the same differentiation capacity in vivo in the tissue recombination assays and hence unlabeled ADM were used with GFP+ mesenchyme in the tissue recombinants as a method of identifying cell lineages.

\section{Tissue Recombination}

All animal experiments were approved by the Institutional Animal Care and Use Committee at the University of Illinois. C57BL/6 mice [C57BL/6-Tg(ACTB-EGFP)1Osb/J] mice heterozygous for enhanced GFP under the control of a chicken $\beta$-actin promoter and cytomegalovirus enhancer (Jackson Laboratory, Bar Harbor, Me., USA) were bred to generate enhanced GFP+ mice. Enhanced GFP+ mice were identified by illumination with a UV lamp and served as hosts for tissue recombinant transplantation and also to produce GFP+ offspring for derivation of fetal/neonatal mesenchyme. Timed pregnant transgenic green $\mathrm{C} 57 \mathrm{BL} / 6$ mice were used to isolate fetal/neonatal mesenchyme. Pregnant mice were sacrificed at 14.5 or 16.5 days of gestation to obtain fetuses that were used as a source of UGS or skin, respectively. Neonatal uteri were obtained from 5-day-old GFP+ females. Uteri, skin, and UGS were incubated with a $1 \%$ trypsin solution in $\mathrm{Ca}^{++}$- and $\mathrm{Mg}^{++}$-free media [Bigsby et al., 1986; Cooke et al., 1997; Simon et al., 2009] to digest the basement membrane between the epithelium and mesenchyme. The UGS was then separated into its connective tissue (UGM) and epithelial (UGS epithelium; UGE) components. UtM and UtE and the dermis and epidermis of the skin were separated by the same procedure.

Mesenchyme from each organ was recombined with homologous epithelium as a control. Tissue recombinants of various mesenchymes and ADM cells were prepared by placing the mesenchyme in a $1.5-\mathrm{ml}$ Eppendorf tube, adding ADM cells, and then centrifuging at $1,000 \mathrm{rpm}$ for $1 \mathrm{~min}$. For tissue recombinations of UGM and ADM cells, one tissue recombinant represented 1 UGM from a 16.5-day fetus recombined with approximately $3 \times 10^{5} \mathrm{ADM}$ cells. For uterine and skin tissue recombinations, mesenchymes (approximately $5 \mathrm{~mm}$ in diameter) were recombined with $3 \times 10^{5}$ ADM cells. Resultant tissue recombinants were placed on nutrient
$1 \%$ agar plates and incubated overnight at $37^{\circ} \mathrm{C}$ in a $5 \% \mathrm{CO}_{2}$ atmosphere [Cooke et al., 1997].

Tissue recombinations were grafted and grown for 4 weeks under the renal capsule of adult syngeneic GFP+ hosts (male hosts for tissue recombinants with UGM, female hosts for those with UtM, and male and female hosts for grafts containing skin mesenchyme) [Cooke et al., 1997; Cunha et al., 2004]. Briefly, hosts were anesthetized with ketamine/xylazine $(80 \mathrm{mg} / \mathrm{kg}$ ketamine and 15 $\mathrm{mg} / \mathrm{kg}$ xylazine i.p). A 2-cm midline incision was made through the skin just posterior to the ribs. Small bilateral incisions were made through the body wall parallel to the long axis of the kidney. Kidneys were exteriorized and small incisions made in the renal capsule, then the tissue recombinants were placed in a pocket created between the renal tissue and the overlying capsule. Kidneys were then returned to the body cavity and muscle and skin were sutured separately. The wild-type ADM and GFP+ mesenchyme tissue recombinants were grafted into GFP hosts to ensure that no host cells would be misidentified as differentiating ADM. Following graft recovery, GFP expression was confirmed in grafts to ensure that the differentiated epithelium was of ADM origin.

To determine the potential of differentiated UtE to respond to its primary mitogen, $17 \beta$-estradiol $\left(\mathrm{E}_{2}\right)$, some female hosts bearing tissue recombinants of UtM plus UtE or UtM plus ADM were ovariectomized 4 weeks after grafting and 2 weeks later were given $125 \mathrm{ng}$ of $\mathrm{E}_{2}$ in $0.1 \mathrm{ml}$ of oil or vehicle alone. Epithelial proliferation was assessed $16 \mathrm{~h}$ later using an antigen identified by monoclonal antibody Ki67 (MKI67) immunohistochemistry and the expression of estrogen receptor 1 (ESR1) was assessed by immunohistochemistry [Cooke et al., 1997]. Expression of progesterone receptor (PR) was assessed in tissue recombinants in which the hosts were treated for 3 days with $125 \mathrm{ng}$ of $\mathrm{E}_{2}$ in $0.1 \mathrm{ml}$ of oil or vehicle alone and harvested $24 \mathrm{~h}$ later, as described [Kurita et al., 2000]. Host uterus was also used as a positive control.

\section{Immunohistochemistry and Immunofluorescence}

Grafts were fixed in 10\% neutral-buffered formalin, dehydrated through graded alcohols, and embedded in paraffin. Grafts were sectioned at $4 \mu \mathrm{m}$, deparaffinized, and rehydrated. To facilitate antigen detection, slides were placed in $10 \mathrm{mM}$ boiling sodium citrate buffer ( $\mathrm{pH}$ 6.0) for $10 \mathrm{~min}$ and then cooled to ambient temperature. Endogenous peroxidase activity was quenched by incubation with $0.3 \% \mathrm{H}_{2} \mathrm{O}_{2}$ for $20 \mathrm{~min}$. Primary antibodies used for immunohistochemistry were anti-mouse MKI67 (BD Biosciences, San Jose, Calif., USA), rabbit polyclonal anti-human PR (Dako, Carpinteria, Calif., USA, A0098, shown to bind PR from mouse tissues in Zeng et al. [2008] and Simon et al. [2009]), and anti-mouse ESR1 (Novocastra Ltd., Newcastle-upon-Tyne, UK). A biotinylated secondary antibody was used for MKI67 and ESR1 and horseradish peroxidase-conjugated antibody was used for PR. The labeling indices of ESR1- and MKi67-positive cells were determined by dividing the number of the respective positive cells by the total number of cells analyzed per section and multiplying by 100 . Data were obtained from 3 sections per tissue recombinant from hosts treated with either $E_{2}$ or oil and at least 300 cells were analyzed per section.

For immunofluorescence, all the procedures followed for immunohistochemistry, except peroxidase quenching, were performed. Primary antibodies used were anti-rabbit GFP (Invitrogen, Carlsbad, Calif., USA), NKX3.1 (Chemicon), and cytokeratin 8 (CK-8; Developmental Studies Hybridoma Bank, University of 
Fig. 1. Stem cell identity of ADM and mesenchyme isolation for tissue recombinants. a Marker profile for the M25.2 clonally derived population of $\mathrm{ADM}$ demonstrates that the majority of cells express Sca-1 and CD90.2, while many cells also express CD34. In contrast, the percentage of cells expressing CD146, CD31, CD13, and CD45 is similar to the low level of nonspecific fluorescence observed in control samples. b M2.1, another clone of ADM, has a similar marker profile, with $95-99 \%$ of cells expressing Sca-1 and CD90.2, and the majority also expressing CD34. c Separation of fetal and neonatal mesenchyme from epithelium for tissue recombination. Following trypsinization and mesenchymal/ epithelial separation, the neonatal uterus (left) can be clearly separated into UtM and UtE. Note that the epithelium is obtained as an entire unbroken fragment, which essentially eliminates residual epithelial contamination of the UtM. Similarly, fetal UGS (right) can be separated into UGM and UGE, respectively, and here again the entire UGE is removed from the UGS as an intact structure.


M25.2
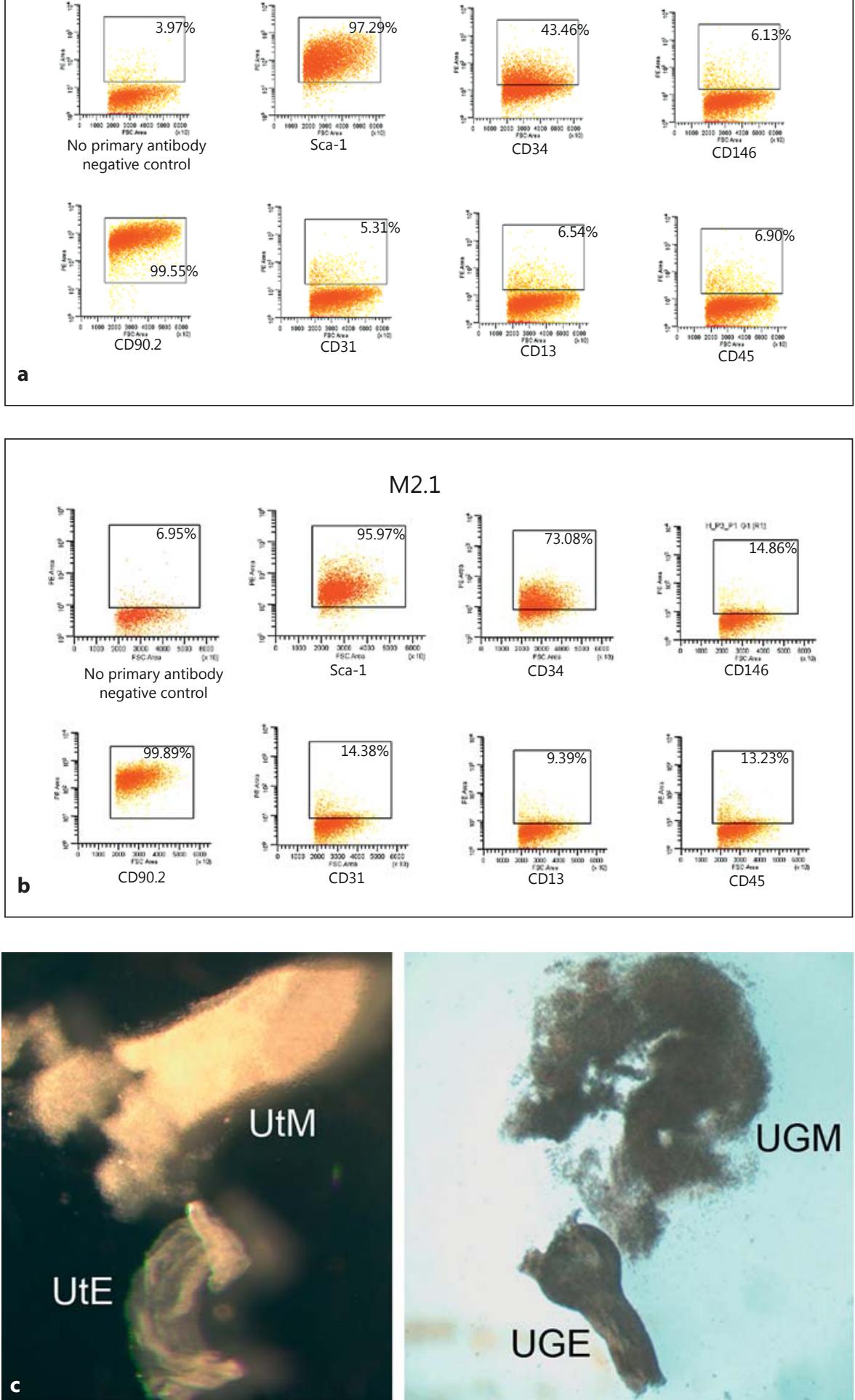

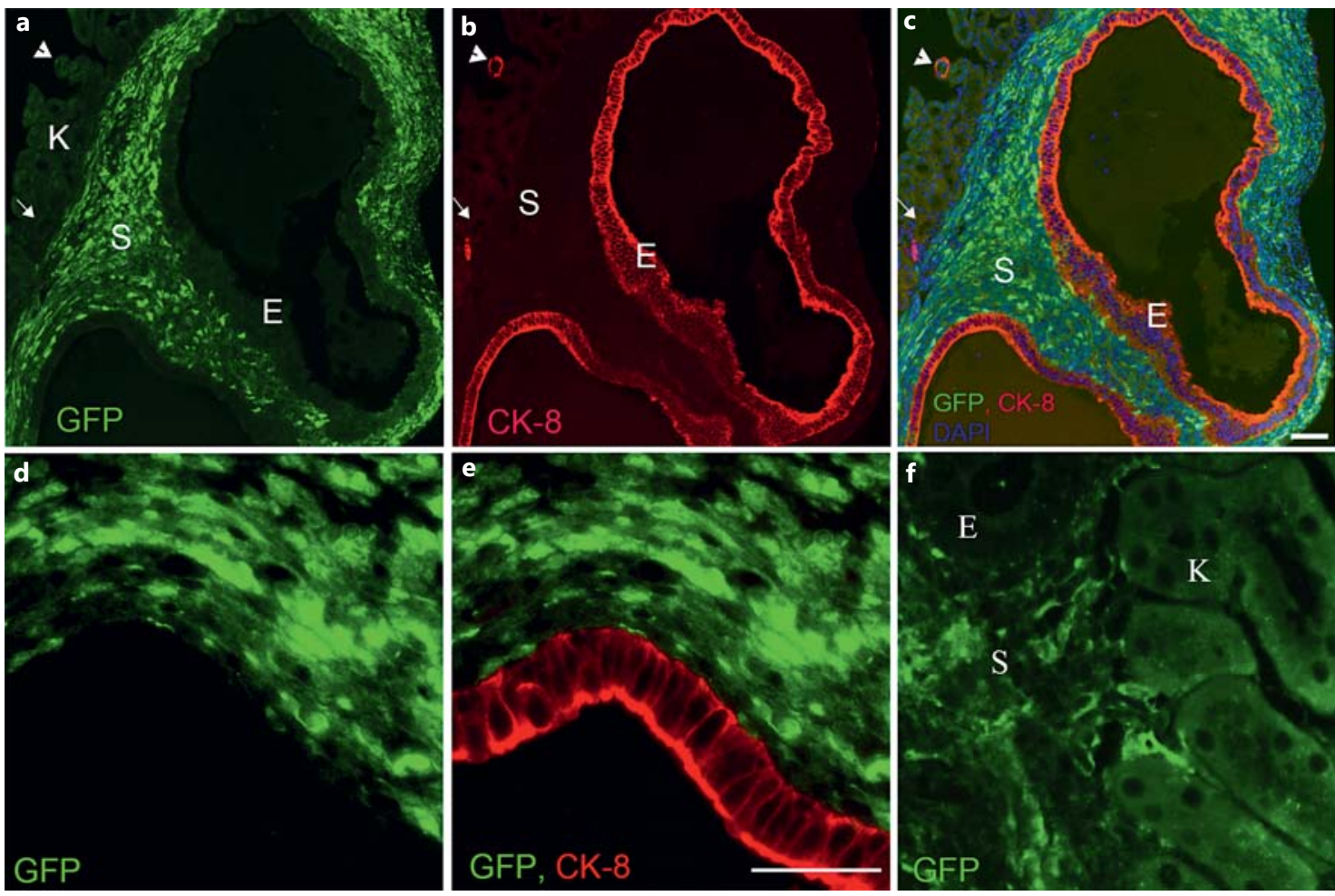

Fig. 2. ADM form UtE when recombined with neonatal UtM in tissue recombinants. a Tissue recombinant of GFP-labeled neonatal UtM (green) and unlabeled ADM. Epithelial cells (E) lack GFP, indicating an ADM origin, while stroma (S) was GFP+. Kidney (K) tissue from the host was visible surrounding recombinants. White arrow indicates proximal convoluted tubules, and the arrowhead loops of Henle. b Immunohistochemical staining of tissue recombinants for CK-8 (red), a marker of UtE, showing CK-8 expression in UtE derived from ADM. Note also the simple columnar epithelium, a hallmark of UtE. $\mathbf{c}$ Merged image of $\mathbf{a}$ and $\mathbf{b}$ demonstrating that the CK-8-labeled epithelium is derived from ADM, while the GFP+ stromal layer does not express the uterine epithelial marker. Blue = DAPI-labeled nuclei. $\mathbf{d}$, e Higher magnifications of tissue recombinants in which the stroma contains GFP+ cells (d), and epithelial cells strongly labeled with CK-8 are comprised of GFP- cells derived from $\operatorname{ADM}(\mathbf{e})$. f Proximal convoluted tubules of the host kidney surrounding the recombinants are visible and contain weak, diffuse GFP, as reported by Ma et al. [2010]. Scale bars $=10 \mu \mathrm{m}$.
Iowa). The secondary antibodies used were fluorescein or tetramethylrhodamine isothiocyanate-conjugated anti-rabbit antibodies. Appropriate negative controls were run in parallel. Slides were mounted with Vectashield containing 4-,6-diamidino2-phenylindole (H-1200; Vector Laboratories, Burlingame, Calif., USA) and photomicrographs were taken with a Retiga 2000R digital camera (QImaging, Surrey, B.C., Canada) mounted on an Olympus BX51 fluorescence microscope (Olympus Corp., Melville, N.Y., USA) using bright-field illumination or appropriate fluorescence excitation and emission filters.

\section{Results}

\section{Tissue Recombinants of Fetal UtM and ADM Form}

Functional UtE

Clonal populations of ADM were analyzed by FACS to verify stem cell marker expression. As previously report- ed, ADM expressed multiple markers associated with stem and progenitor cells [Wang et al., 2012]. Greater than $95 \%$ of both clonal populations expressed Sca- 1 and CD90.2, and $43 \%$ of M25.2 cells and 73\% of M2.1 cells also expressed CD34 (fig. 1a, b). Also consistent with our previous report, fewer than $15 \%$ of cells expressed CD146 and CD13, markers associated with pericytes and mesenchymal stem cells [Covas et al., 2005, 2008; Crisan et al., 2008; da Silva Meirelles et al., 2008]. Less than 15\% of ADM expressed endothelial markers CD31 and CD45 [Crisan et al., 2008; Tárnok et al., 2010].

To establish whether fetal mesenchyme could induce $\mathrm{ADM}$ to differentiate into UtE, neonatal GFP+ UtM was recombined with $\mathrm{ADM}$ and grafted into GFP+ hosts. Fetal stroma was separated from epithelium using an enzymatic separation technique developed by our group, in which only $2-3 \%$ of UtM or UGM tissue prepared using 

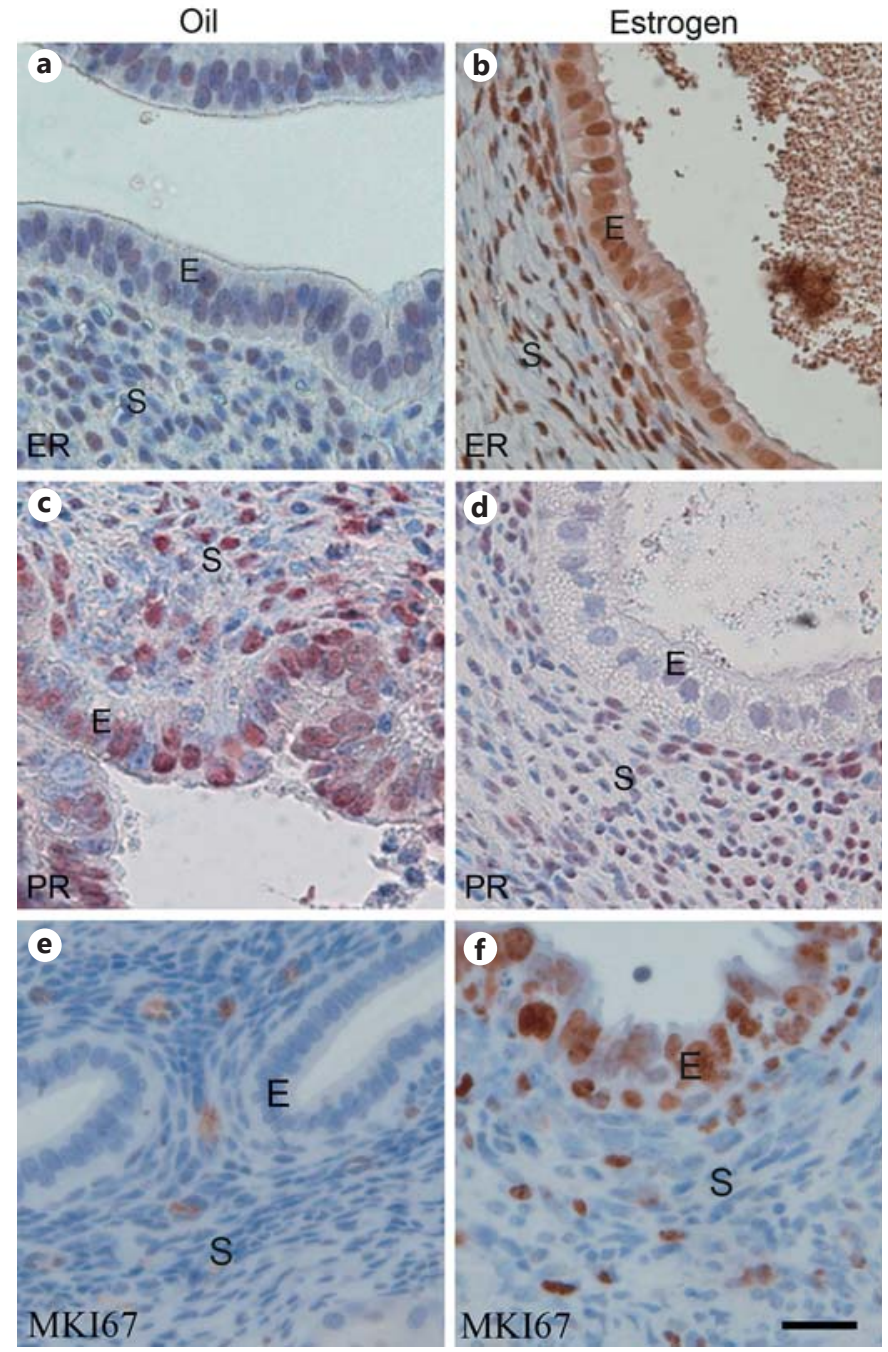

Fig. 3. Epithelium in tissue recombinants of neonatal UtM plus $\mathrm{ADM}$ is responsive to estrogen. $\mathbf{a}, \mathbf{b}$ ESR 1 is upregulated in the $\mathrm{ADM}$-derived uterine epithelial layer $(\mathrm{E})$ and surrounding uterine stroma $(\mathrm{S})$ in grafts from $\mathrm{E}_{2}$-treated (b) compared to oil-treated ovariectomized hosts (a). c, d PR is expressed in both epithelium and stroma of neonatal UtM plus ADM tissue recombinants in oiltreated ovariectomized mice (c), but is downregulated specifically in UtE following treatment of ovariectomized host mice with $\mathrm{E}_{2}$ (d). e UtE in tissue recombinants of neonatal UtM plus ADM shows minimal expression of MKI67 in ovariectomized mice treated with oil only. $\mathbf{f}$ Administration of $\mathrm{E}_{2}$ to ovariectomized hosts bearing neonatal UtM plus ADM tissue recombinants causes proliferation (MKI67 expression) in UtE of the tissue recombinants. Scale bar $=10 \mu \mathrm{m}$.

this technique contains residual epithelial tissue [Bigsby et al., 1986] (fig. 1c). Eight of 17 recovered grafts contained a mixture of well-organized stroma and simple columnar epithelium (fig. 2). Epithelium in GFP+ UtM and ADM tissue recombinants did not express GFP, indicat- ing derivation from $\mathrm{ADM}$ (fig. 2a, c-f). This epithelium, but not surrounding stroma, expressed CK-8, a marker of UtE (fig. 2b, c, e). Some GFP- cells were also present in the stroma of recombinants (fig. $2 \mathrm{f}$ ) but did not have epithelial morphology and did not express CK-8.

Proximal convoluted tubules from the kidney of the host were observed surrounding the tissue recombinant (fig. 2a-c, f). These proximal tubules were identifiable due to their cuboidal epithelium, which contained a brush border of microvilli, and did not express CK-8 [Piepenhagen and Nelson, 1995; Luis Reyes et al., 2002]. Although proximal tubules constituted the majority of tubules in the renal cortex adjacent to the tissue recombinants, some loops of Henle containing squamous cells expressing CK-8 are also seen (fig. 2b, c). Expression of GFP was diffuse and weak in cells of the kidney tubules, as reported previously in these enhanced GFP mice by Ma et al. [2010] (fig. 2f).

UtE from ovariectomized mice showed robust mitogenesis and downregulation of PR in response to estrogen, both of which are hallmarks of the normal uterine response to estrogenic stimulation. Epithelial cells in UtM and ADM tissue recombinants in oil-treated hosts showed low basal levels of ESR1, with $12.8 \pm 2.2 \%$ of cells expressing the receptor, which is not expressed in ADM (fig. 3a). However, epithelial cells exhibited strong expression of ESR1, as expected, in hosts treated with $\mathrm{E}_{2}$ (fig. $3 \mathrm{~b}$ ), with $88.2 \pm 9.2 \%$ of cells containing the receptor. Epithelial cells in these tissue recombinants also showed a pronounced mitogenic response to $E_{2}$, with $91.4 \pm 5.8 \%$ of cells expressing MKI67, a marker of cell proliferation, following $E_{2}$ treatment. Conversely, epithelium of tissue recombinants in hosts injected with oil vehicle showed minimal proliferative activity, with $3.3 \pm 1.0 \%$ of cells expressing MKI67 (fig. 3e, f). Epithelium in UtM and ADM tissue recombinants showed downregulation of $P R$ in response to $E_{2}$, while PR expression remained high in epithelium of tissue recombinants from oil-treated hosts (fig. 3c, d).

\section{Tissue Recombination of UGM and ADM Does Not \\ Induce Development of Prostatic Epithelium}

To determine whether ADM could differentiate into cells of endodermal origin, we recombined UGM with $\mathrm{ADM}$ and determined whether ADM could form prostatic epithelium during a 4 -week grafting period. As a positive control, UGM from GFP+ mice was recombined with fetal UGE from wild-type mice lacking GFP. Control recombinants yielded grafts containing unlabeled (GFP-) prostatic epithelium surrounded by GFP+ stroma (fig. 4a). Nine UGM and ADM tissue recombinants 

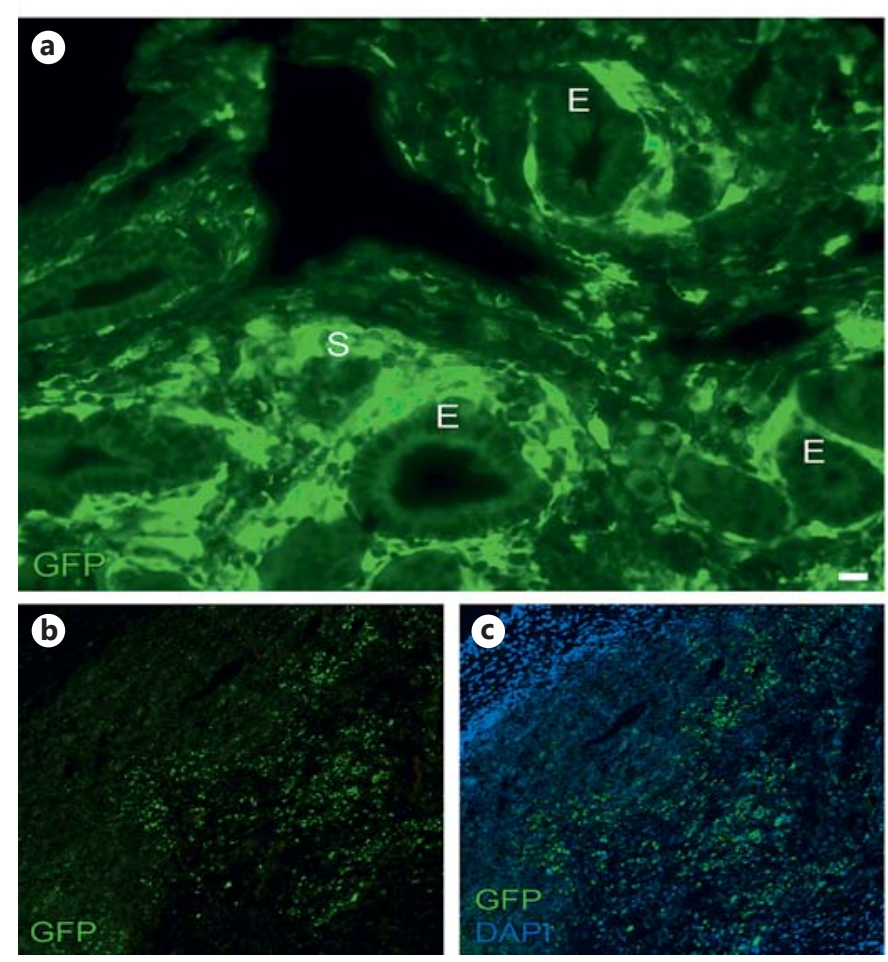

Fig. 4. ADM do not differentiate into prostatic epithelium when recombined with fetal UGM and grown as tissue recombinants under the renal capsule of male host mice. a In tissue recombinants of fetal UGM from GFP+ mice and fetal UGE from wild-type mice, epithelial prostatic ducts similar to prostate in vivo were formed; the prostatic stroma (S), but not epithelium (E), expressed GFP. b-d tissue recombinants composed of fetal UGM from GFP mice and unlabeled ADM. ADM could be identified in recovered tissue recombinants, along with GFP-labeled stromal cells, but prostatic epithelium was not observed and ADM cells in the graft were disorganized, in contrast to the extensive prostatic epithelium seen in

were grafted into hosts and 6 were recovered. In contrast to results obtained with UtM, none contained epithelium with a simple columnar prostatic phenotype (fig. $4 \mathrm{~b}-\mathrm{f}$ ). Unlabeled ADM were present in a disorganized pattern in recovered grafts and had not integrated with GFP-labeled cells from UGM (fig. 4b-f). No organized tissue or recognizable structure was observed within the recombinants, such as vessels, nerves, or skeletal myotubes, which ADM have been reported to form following transplantation into skeletal and cardiac muscle [Berry et al., 2007; Chun et al., 2013].

Mesoangioblasts Differentiate into

Uterine Epithelium
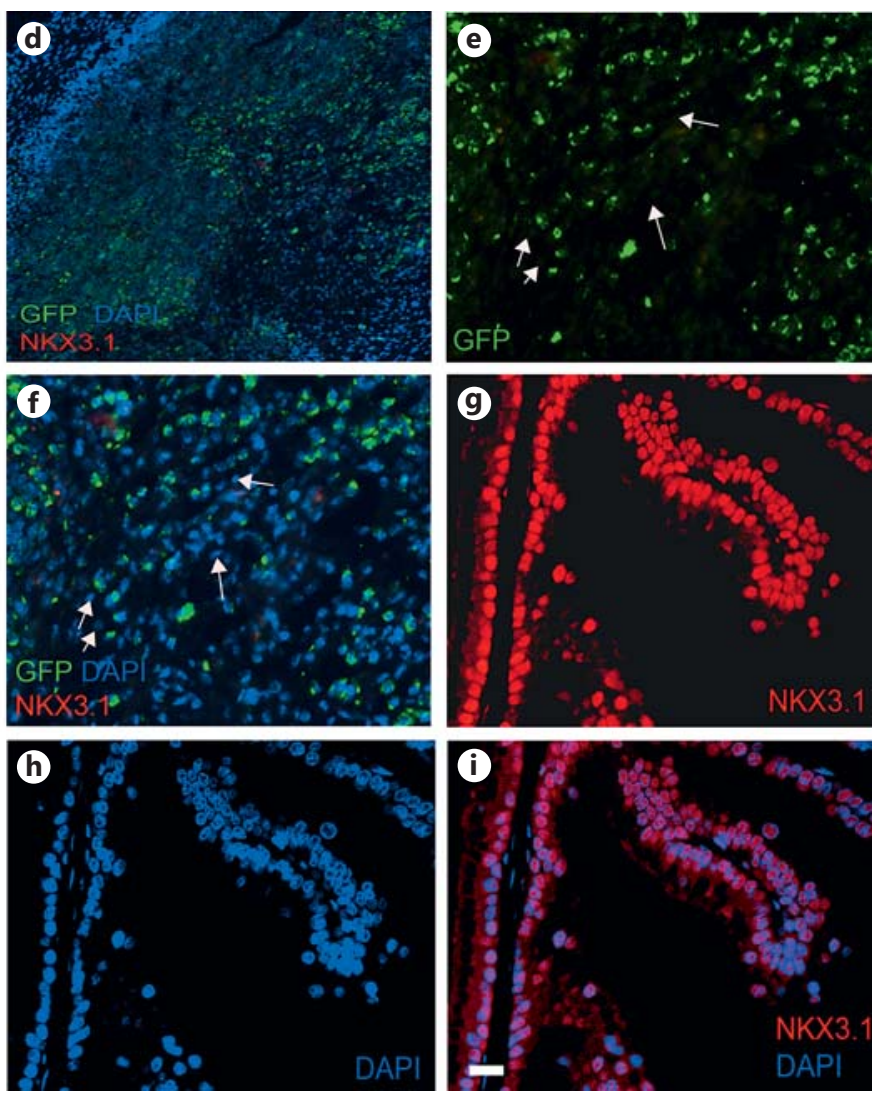

control UGM \pm UGE grafts (a). d Consistent with the lack of identifiable prostatic epithelium, unlabeled ADM in grafts of UGM plus ADM tissue recombinants do not express the prostate epithelial marker NKX3.1. c, d DAPI-labeled nuclei (blue). e, f Higher magnification images of the recovered grafts, in which both GFP+ and GFP- cells are present, but GFP- cells (white arrows) do not express NKX3.1 in their nuclei. g-i In contrast, control UGM \pm UGE grafts show strong nuclear immunostaining for NKX3.1 (red; $\mathbf{g}, \mathbf{i})$ in the prostatic ducts. $\mathbf{h}$ DAPI-stained nuclei of the prostate shown in $\mathbf{g}$. $\mathbf{i}$ Merged image of $\mathbf{g}$ and $\mathbf{h}$.

NKX3.1 is a transcription factor present specifically in adult prostate epithelial cells [Bieberich et al., 1996; Sciavolino et al., 1997]. Using prostate as a positive control, we observed NKX3.1 immunostaining in the nuclei of prostate epithelial cells, as expected (fig. $4 \mathrm{~g}-\mathrm{i}$ ), but not in GFP- cells in $\mathrm{UGM} \pm \mathrm{ADM}$ recombined tissue (fig. $4 \mathrm{~b}-\mathrm{f}$ ), indicating that prostatic epithelium was not present.

Tissue Recombination of Fetal Dermis and ADM Does

Not Induce Epidermal Development

Tissue recombinants of fetal dermis and either fetal epidermis as a control or ADM were prepared to test the

Cells Tissues Organs 2013;198:169-178 


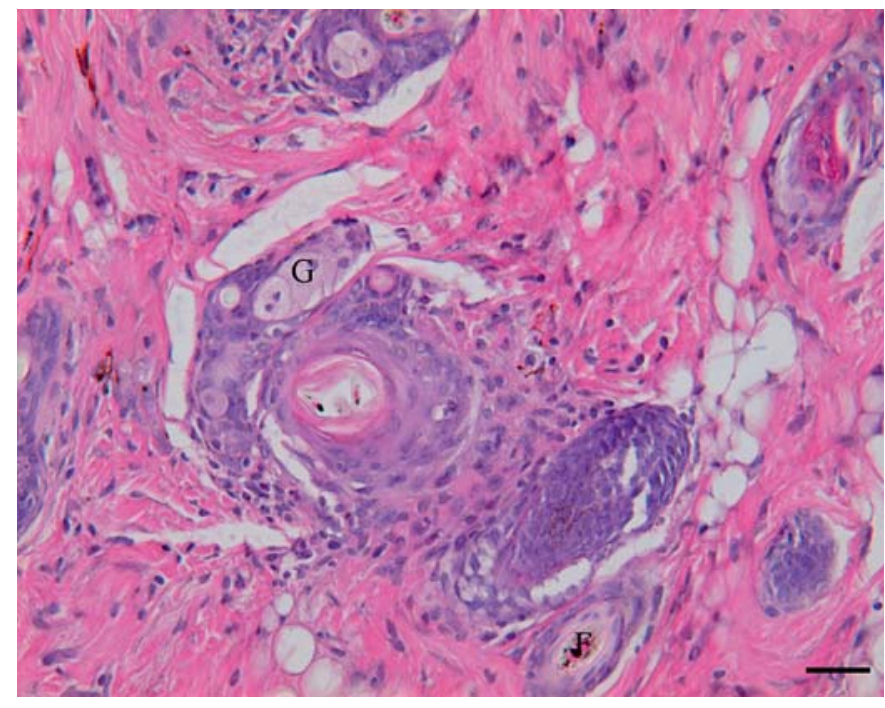

Fig. 5. Tissue recombinants of mesenchyme and epithelium from fetal skin yielded epithelium typical of epidermis. Hair follicles (F) and sebaceous glands $(\mathrm{G})$ were present in control tissue recombinants. Scale bar $=10 \mu \mathrm{m}$.

hypothesis that $\mathrm{ADM}$ can differentiate into epidermis, an ectodermal derivative, in response to instructive induction from the dermis. Three separate replicates were completed, with at least 4 tissue recombinants in each replicate, or a total of 12 replicates. Control tissue recombinants of dermis plus fetal epidermis contained an epidermal layer, complete with hair follicles and sebaceous glands (fig. 5). However, tissue recombinants of fetal dermis and ADM did not yield organized or identifiable structures (data not shown), indicating that ADM could not be programmed to differentiate into epidermis, as ectodermal derivative, by fetal dermis.

\section{Discussion}

Mesoangioblasts are vessel-derived stem cells that can be induced to differentiate into mesodermal derivatives, including smooth, skeletal, and cardiac muscle, and adipocytes [Minasi et al., 2002; Sampaolesi et al., 2006; Berry et al., 2007; Odintsov et al., 2011; Wang et al., 2012; Chun et al., 2013]. Reports of postnatal mesoangioblasts derived from bone marrow, atria and ventricles of the heart, aorta, and skeletal muscle have focused primarily on their myogenic potential. The capacity of postnatal mesoangioblasts to regenerate skeletal muscle in animal models of degenerative muscle diseases in these reports has led to a phase I clinical trial in Duchenne patients, highlighting their therapeutic potential for neurodegenerative diseases. In addition to their myogenic potential, we report here that postnatal mesoangioblasts differentiate into epithelial cells of the reproductive system. ADM differentiated into UtE when recombined with UtM. The differentiated cells had the typical simple columnar uterine epithelial phenotype and expressed CK-8, a marker of UtE.

Estrogen is the major endocrine regulator of uterine epithelial cells, and $\mathrm{E}_{2}$ acts through ESR1 to control uterine epithelial morphogenesis, proliferation, cytodifferentiation, and secretory activity. Our results indicate that ADM cells recombined with UtM were not only morphologically and histologically identical to uterine epithelial cells following the grafting period, but also responded to $\mathrm{E}_{2}$, as evidenced by increased epithelial proliferation, upregulation of epithelial ESR1 expression, and downregulation of uterine epithelial PR. This indicates that the UtE derived from ADM expressed normal functional characteristics of this tissue. UtE is of mesodermal origin, and these data are therefore consistent with previous findings by our group and others that ADM can differentiate into mesodermal derivatives, including smooth, skeletal, and cardiac muscle, osteoblasts, chondrocytes, adipocytes, and blood cells [Minasi et al., 2002; Sampaolesi et al., 2006; Berry et al., 2007; Odintsov et al., 2011; Chun et al., 2013] and indicate that ADM have broad developmental potential within the mesodermal lineage.

Our present results also indicate that although ADM are developmentally plastic and can give rise to tissues of mesodermal origin they do not normally form, such as UtE, these cells may be predominantly restricted to the generation of mesodermally derived structures. We did not observe reprogramming of $\mathrm{ADM}$ into epidermis, a tissue of ectodermal origin, when recombined with fetal dermis, despite our previous observations that ADM differentiate into oligodendrocytes and Schwann cells of ectodermal origin in the central and peripheral nervous system, respectively [Berry et al., 2007; Wang et al., 2012]. It is likely that ADM maintain neural differentiation potential as a result of the similarity in signaling pathways necessary for neural and muscle specification. There are numerous precedents for adult stem cells to differentiate into both neural and muscle cells. For example, stem cells from the adult brain and from the neural tube differentiate into muscle cells [Tajbakhsh et al., 1994; Galli et al., 2000], and bone marrow cells and some muscle stem cells differentiate into both neural and muscle cells [Ferrari et al., 1998; Brazelton et al., 2000; Qu-Peterson et al., 2002; Tamaki et al., 2005]. 
Similar to the results with instructive fetal ectodermal mesenchyme, tissue recombinants of ADM plus fetal UGM failed to direct postnatal ADM to become prostate epithelium, an endodermal derivative. This is consistent with a previous report in avians, in which murine mesoangioblasts from embryonic dorsal aorta were transplanted into quail and chick embryos, and failed to differentiate into cells of endodermal origin [Minasi et al., 2002].

The results of our tissue recombination study with ADM contrast with those from our previous report using tissue recombinants to test the potential of SSC to differentiate into various lineages. SSC were capable of differentiating into mesodermal, ectodermal, and endodermal derivatives by the same instructive fetal mesenchymes used in this study [Simon et al., 2009]. The contrasting results between the tissue recombination studies with SSC and ADM highlight the differing potential of these two postnatal stem cell populations as well as the utility of tissue recombination to explore differentiation of stem cells. Using this method, we demonstrated that SSC have a pluripotent nature, similar to embryonic stem cells. This conclusion has important clinical implications, given the limited availability of embryonic stem cells and controversy surrounding their use. In contrast, we have shown that ADM are largely restricted to generating mesodermal derivatives, and that they have the capacity to differentiate into functional mesodermal cells in response to instructive microenvironments which differ from those that mesoangioblasts would encounter in their native vascular niche.

The broad mesodermal potential of ADM, together with their appropriate developmental response to cues in their environment, indicates they are good candidate stem cells for regenerative medicine applications. Tissue regeneration and engineering frequently require the simultaneous generation or replacement of many complex structures, including nerve, muscle, vasculature, and bone. Given our results here and in previous reports, transplantation of undifferentiated ADM into severely damaged tissue or an engineered scaffold with appropriate growth factors in defined regions may lead to the generation or regeneration of multiple structures and cells types at the same time, depending upon the surrounding microenvironments.

\section{Acknowledgments}

We gratefully acknowledge Janie Frye from the Department of Pathobiology at the College of Veterinary Medicine, University of Illinois at Urbana-Champaign, for her assistance in FACS analysis of surface markers on mesoangioblasts, and Dr. Federico Zuckerman, also from the Department of Pathobiology of the College of Veterinary Medicine, University of Illinois, for use of the iCyte cell sorter in his laboratory.

\section{References}

Berry, S.E., J. Liu, E.J. Chaney, S.J. Kaufman (2007) Multipotential mesoangioblast stem cell therapy in the $\mathrm{mdx} / \mathrm{utrn}^{-/-}$mouse model for Duchenne muscular dystrophy. Regen Med 2: 275-288.

Bieberich, C. J., K. Fujita, W.W. He, G. Jay (1996) Prostate-specific and androgen-dependent expression of a novel homeobox gene. J Biol Chem 271: 31779-31782.

Bigsby, R.M., P.S. Cooke, G.R. Cunha (1986) A simple efficient method for separating murine uterine epithelial and mesenchymal cells. Am J Physiol 251: E630-E636.

Brazelton, T.R., F.M. Rossi, G.I. Keshet, H.M. Blau (2000) From marrow to brain: expression of neuronal phenotypes in adult mice. Science 290: 1775-1779.

-Chun, J., R. O’Brien, M. Song, B.F. Wondrasch, S.E. Berry (2013) Injection of vessel-derived stem cells prevents dilated cardiomyopathy and promotes angiogenesis and endogenous cardiac stem cell proliferation in $m d x / \mathrm{trn}^{-/-}$ but not aged $m d x$ mouse models for $\mathrm{Du}$ chenne muscular dystrophy. Stem Cells Transl Med 2: 68-80.

\begin{abstract}
Cooke, P.S., D.L. Buchanan, P. Young, T. Se- Crisan, M., S. Yap, L. Casteilla, C. Chen, M. Cortiawan, J. Brody, J. Taylor, K.S. Korach, D.B. Lubahn, G.R. Cunha (1997) Stromal estrogen receptors (ER) mediate the mitogenic effect of estradiol on uterine epithelium. Proc Natl Acad Sci USA 94: 6535-6540.

Covas, D.T., C.E. Piccinato, M.D. Orellana, J.L. Siufi, W.A. Silva, Jr., R. Proto-Siqueira, E.G. Rizzatti, L. Neder, A. Silva, V. Rocha, M. Zago (2005) Mesenchymal stem cells can be obtained from the human saphena vein. Exp Cell Res 309: 340-344.

Covas, D.T., R.A. Panepucci, A.M. Fontes, W.A. Silva, Jr., M.D. Orellana, M.C. Freitas, L. Neder, A.R. Santos, L.C. Peres, M.C. Jamur, M.A. Zago (2008) Multipotent mesenchymal stromal cells obtained from diverse human tissues share functional properties and geneexpression profile with CD146 \pm perivascular cells and fibroblasts. Exp Hematol 36: 642654 . selli, T.S. Park, G. Andriolo, B. Sun, B. Zheng, L. Zhang, C. Norotte, P.N. Teng, J. Traas, R. Schugar, B.M. Deasy, S. Badylak, H.J. Buhring, J.P. Giacobino, L. Lazzari, J. Huard, B. Péault (2008) A perivascular origin for mesenchymal stem cells in multiple human organs. Cell Stem Cell 3: 301-313.

Cunha, G.R., A.A. Donjacour, P.S. Cooke, S. Mee, R.M. Bigsby, S.J. Higgins, Y. Sugimura (1987) The endocrinology and developmental biology of the prostate. Endocr Rev 8: 338-362.

Cunha, G.R., P.S. Cooke, T. Kurita (2004) Role of stromal-epithelial interactions in hormonal responses. Arch Histol Cytol 67: 417-434.

da Silva Meirelles, L., A.I. Caplan, N.B. Nardi (2008) In search of the in vivo identity of mesenchymal stem cells. Stem Cells 26: 2287-2299.

De Angelis, L., L. Berghella, M. Coletta, L. Lattanzi, M. Zanchi, M.G.Cusella-De Angelis, C. Ponzetto, G. Cossu (1999) Skeletal myogenic progenitors originating from embryonic dorsal aorta coexpress endothelial and myogenic markers and contribute to postnatal muscle growth and regeneration. J Cell Biol 147: 869-877.
\end{abstract}

Mesoangioblasts Differentiate into

Uterine Epithelium
Cells Tissues Organs 2013;198:169-178 DOI: $10.1159 / 000354900$ 
Ferrari, G., G. Cusella-De Angelis, M. Coletta, E. Paolucci, A. Stornaiuolo, G. Cossu, F. Mavilio (1998) Muscle regeneration by bone marrowderived myogenic progenitors. Science 279: 1528-1530.

Galli, R., U. Borello, A. Gritti, M.G. Minasi, C. Bjornson, M. Coletta, M. Mora, M.G.C. De Angelis, R. Fiocco, G. Cossu, A.L. Vescovi. (2000) Skeletal myogenic potential of human and mouse neural stem cells. Nature Neuroscience 3: 986-991.

-Galvez, B.G., M. Sampaolesi, A. Barbuti, A. Crespi, D. Covarello, S. Brunelli, A. Dellavalle, S. Crippa, G. Balconi, I. Cuccovillo, F. Molla, L. Staszewsky, R. Latini, D. DiFrancesco, G. Cossu (2008) Cardiac mesoangioblasts are committed, self-renewable progenitors, associated with small vessels of juvenile mouse ventricle. Cell Death Differ 15: 1417-1428.

Greenwood, H.L., P.A. Singer, G.P. Downey, D.K. Martin, H. Thorsteinsdóttir, A.S. Daar (2006) Regenerative medicine and the developing world. PLoS Med 3: 1496-1500.

Kurita, T., K.J. Lee, P.S. Cooke, J.A. Taylor, D.B. Lubahn, G.R. Cunha (2000) Paracrine regulation of epithelial progesterone receptor by estradiol in the mouse female reproductive tract. Biol Reprod 62: 821-830.

Kurita, T., P.S. Cooke, G.R. Cunha (2001) Epithelial-stromal tissue interaction in paramesonephric (Müllerian) epithelial differentiation. Dev Biol 240: 194-211.

Luis Reyes, J., M. Lamas, D. Martin, M. del Carmen Namorado, S. Islas, J. Luna, M. Tauc, L. González-Mariscal (2002) The renal segmental distribution of claudins changes with development. Kidney Int 62: 476-487.

Ma, DF, H. Tezuka, T. Kondo, K. Sudo, D.F. Niu, T. Nakazawa, T. Kawasaki, T. Yamane, N. Nakamura, R. Katoh (2010) Differential tissue expression of enhanced green fluorescent protein in 'green mice'. Histol Histopathol 25: 749-754.
Minasi, M.G., M. Riminucci, L. De Angelis, U. Borello, B. Berarducci, A. Innocenzi, A. Caprioli, D. Sirabella, M. Baiocchi, R. De Maria, R. Boratto, T. Jaffredo, V. Broccoli, P. Bianco, G. Cossu (2002) The meso-angioblast: a multipotent, self-renewing cell that originates from the dorsal aorta and differentiates into most mesodermal tissues. Development 129: 2773-2783.

Morosetti, R., M. Mirabella, C. Gliubizzi, A. Broccolini, L. De Angelis, E. Tagliafico, M. Sampaolesi, T. Gidaro, M. Papacci, E. Roncaglia, S. Rutella, S. Ferrari, P.A. Tonali, E. Ricci, G. Cossu (2006) MyoD expression restores defective myogenic differentiation of human mesoangioblasts from inclusion-body myositis muscle. Proc Natl Acad Sci USA 103: 16995-17000.

Odintsov, B., J. Chun, J.A. Mulligan, S.E. Berry (2011) $14.1 \mathrm{~T}$ whole body MRI for detection of mesoangioblast stem cells in a murine model of Duchenne muscular dystrophy. Magn Reson Med 66: 1704-1714.

Palumbo, R., M. Sampaolesi, F. De Marchis, R. Tonlorenzi, S. Colombetti, A. Mondino, G. Cossu, M.E. Bianchi (2004) Extracellular HMGB1, a signal of tissue damage, induces mesoangioblast migration and proliferation. J Cell Biol 164: 441-449.

Piepenhagen, P.A., W.J. Nelson (1995) Differential expression of cell-cell and cell-substratum adhesion proteins along the kidney nephron. Am J Physiol Cell Physiol 269: C1433-C1449.

Qu-Peterson, Z., B. Deasy, R. Jankowski, M. Ikezawa, J. Cummins, R. Pruchnic, J. Mytinger, B. Cao, C. Gates, A. Wernig, J. Huard (2002) Identification of a novel population of muscle stem cells in mice: potential for muscle regeneration. J Cell Biol 157: 851864.

Sampaolesi, M., S. Blot, G. D’Antona, N. Granger, R. Tonlorenzi, A. Innocenzi, P. Mognol, J. Thibaud, B.G. Galvez, I. Barthélémy, L. Perani, S. Mantero, M. Guttinger, O. Pansarasa, C. Rinaldi, M.G.C. De Angelis, Y. Torrente, C. Bordignon, R. Bottinelli, G. Cossu (2006) Mesoangioblast stem cells ameliorate muscle function in dystrophic dogs. Nature 444: 574579.
Sciavolino, P.J., E.W. Abrams, L. Yang, L.P. Austenberg, M.M. Shen, C. Abate-Shen (1997) Tissue-specific expression of murine Nkx3.1 in the male urogenital system. Dev Dyn 209: 127-138.

Simon, L., G.C. Ekman, N. Kostereva, Z. Zhang, R.A. Hess, M.C. Hofmann, P.S. Cooke (2009) Direct transdifferentiation of stem/progenitor spermatogonia into reproductive and non-reproductive tissues of all germ layers. Stem Cells 27: 1666-1675.

-Tagliafico, E., S. Brunelli, A. Bergamaschi, L. De Angelis, R. Scardigli, D. Galli, R. Battini, P. Bianco, S. Ferrari, G. Cossu, S. Ferrari (2004) TGF $\beta / B M P$ activate the smooth muscle/bone differentiation programs in mesoangioblasts. J Cell Sci 117: 4377-4388.

Tajbakhsh, S., E. Vivarelli, G. Cusella-De Angelis, D. Rocancourt, M. Buckingham, G. Cossu (1994) A population of myogenic cells derived from the mouse neural tube. Neuron 13 : 813-821.

- Tamaki, T., Y. Uchiyama, Y. Okada, T. Ishikawa, M. Sato, A. Akatsuka, T. Asahara (2005) Functional recovery of damaged skeletal muscle through synchronized vasculogenesis, myogenesis, and neurogenesis by muscle-derived stem cells. Circulation 112: 2857-2866.

Tárnok, A, H. Ulrich, J. Bocsi (2010) Phenotypes of stem cells from diverse origin. Cytometry A 77: 6-10.

Wang, L., A. Kamath, J. Frye, G.A. Iwamoto, J. Chun, S.E. Berry (2012) Aorta-derived mesoangioblasts differentiate into oligodendrocytes by inhibition of the Rho kinase signaling pathway. Stem Cells Dev 21: 10691089.

Zeng, Z., M.C. Velarde, F.A. Simmen, R.C.M. Simmen (2008) Delayed parturition and altered myometrial progesterone receptor isoform A expression in mice null for Krüppel-like factor 9. Biol Reprod 78: 10291037. 